\title{
LITERATURE UPDATE FOR TEXAS FLESHY BASIDIOMYCOTA WITH NEW VOUCHERED RECORDS FOR SOUTHEAST TEXAS
}

\author{
David P. Lewis \\ 262 CR 3062 \\ Newton, Texas 75966, U.S.A. \\ dandplewis@gmail.com
}

\author{
Clark L. Ovrebo \\ Department of Biology \\ University of Central Oklahoma \\ Edmond, Oklahoma 73034, U.S.A. \\ covrebo@uco.edu
}

\author{
N. Jay Justice \\ 16055 Michelle Drive \\ Alexander, Arkansas 72002, U.S.A. \\ justice@aristotle.net
}

\begin{abstract}
This is a second paper documenting the literature records for Texas fleshy basidiomycetous fungi and includes both older literature and recently published papers. We report 80 literature articles which include 14 new taxa described from Texas. We also report on 120 new records of fleshy basdiomycetous fungi collected primarily from southeast Texas.
\end{abstract}

RESUMEN

Este es un segundo artículo que documenta el registro de nuevas especies de hongos carnosos basidiomicetos, incluyendo artículos antiguos y recientes. Reportamos 80 artículos científicamente relacionados con estas especies que incluyen 14 taxones con holotipos en Texas. Así mismo, reportamos unos 120 nuevos registros de hongos carnosos basidiomicetos recolectados primordialmente en al sureste de Texas.

PART I-MYCOLOGICAL LITERATURE ON TEXAS FLESHY BASIDIOMYCOTA

Lewis and Ovrebo (2009) previously reported on literature for Texas fleshy Basidiomycota and also listed new vouchered records for Texas of that group. Presented here is an update to the listing which includes literature published since 2009 and also includes older references that we previously had not uncovered. The authors' primary research interests center around gilled mushrooms and boletes so perhaps the list that follows is most complete for the fungi of these groups. We have, however, attempted to locate references for all fleshy basidiomycetous fungi. For a reference to be included in the list, a collection must be cited from Texas. For modern references this is not difficult to discern because of the exacting requirements of locality information and herbarium designations. Not included are publications that give anecdotal records such as "distribution as far as Texas" or something similar without documentation of collections. Liberty is yielded to the literature of the nineteenth or early twentieth centuries where geographic information might not be as complete, nor is there a guarantee that a herbarium specimen exists. Following each reference is a bolded number in parenthesis. This number indicates how many Texas records are included in the article. For taxa that have the holotype from Texas, the binomials and localities (sometimes only the state name was given in the protologue) where collected are provided beneath the reference, followed by the herbarium location of the holotype and following the semicolon is the barcode. We have relied on literature references and online herbaria data (e.g., mycoportal. org) to determine the location of the holotypes. For the older holotypes it is possibly that they have since been nomenclaturally or taxonomically synonymized, but we feel that for the purposes of this paper it is best to give the basionym.

\section{RESULTS}

Adamíčk, S. \& B. Buyck. 2010. Re-instatement of Russula levyana Murrill as a good and distinct American species of Russula section Xerampelinae. Cryptog. Mycol. 31(2):119-135. (1)

Altes, A. \& G. Moreno. 1999. Notes on type materials of Tulostoma (Tulostomataceae): T. macrosporum, T. meridionale and T. utahense. Persoonia 17(2):259-264. (1)

Baroni, T.J. 1992. Clitopilus argentinus (Entolomataceae) in North America II. Persoonia 14(4):361-368. (1)

Baroni, T.J. \& P.B. Matheny. 2011. A re-evaluation of gasteroid and cyphelloid species of Entolomataceae from eastern North America. Harvard Pap. Bot. 16(2):293-310. (1)

J. Bot. Res. Inst. Texas 14(2): $465-479.2020$ 
Baxter, D.V. 1943. Some resupinate polypores from the region of the Great Lakes. XIV. Pap. Michigan Acad. Sci. 28:215233. (1)

Berkeley, M.J. 1873. Notices of North American fungi. Grevillea 2:17-20. (1)

Berkeley, M.J. 1875. Notices of North American fungi. Grevillea 3(28):145-160. (1)

Berkeley, M.J. \& M.A. Curtis. 1853. XLI-Centuries of North American fungi. Ann. Mag. Nat. Hist. Ser. 2, 12:417-435. (1)

Montagnites candollei var. texensis Berk. \& M.A. Curtis (HOLOTYPE: TEXAS. Curtis 3917, herbarium unknown)

Berkeley, M.J. \& M.A. Curtis. 1868. Fungi Cubenses (Hymenomycetes). On Cuban Fungi. J. Linn. Soc., Bot 10 (no. 45-46):280-341. (4)

Both, E.E. \& B. Ortiz-Santana. 2012. An annotated index to species and intraspecific taxa of Boletes (Mycota: Boletales: Boletaceae). Bull. Buffalo Soc. Nat. Sci. 41:3-6. (1)

Burdsall, H.H. 1971. Notes on some lignicolous basidiomycetes of the southeastern United States. J. Elisha Mitchell Sci. Soc. 87:239-245. (1)

Burdsall, H.H. Jr. 1985. A contribution to the taxonomy of the genus Phanerochaete (Corticiaceae, Aphyllophorales). Mycol. Mem. 10:1-165. (1)

Burdsall, H.H. \& K.K. Nakasome. 1978. Taxonomy of Phanerochaete chrysorhizon and Hydnum omnivorum. Mycotaxon 7(1):10-22. (1)

Burdsall, H.H., Jr. \& M.T. Bank. 2001. The genus Laetiporus in North America. Harvard Pap. Bot. 6(1):43-55. (1)

Buyck, B., D. Lewis, G. Eyssartier, \& V. Hofstetter. 2010. Cantharellus quercophilus sp. nov. and its comparison to other small, yellow or brown American chanterelles. Cryptog. Mycol. 31(1):17-33. (4)

Cantharellus quercophilus Buyck \& Lewis (HOLOTYPE: TEXAS. Burleson Co.: Caldwell; PC; MNHN-PC-PC0084726)

Buyck. B. \& V. Hofstetter. 2011. The contribution of tef-1 sequences to species delimitation in the Cantharellus cibarius complex in the southeastern USA. Fungal Diversity 49(1):35-46. (4)

Cantharellus altipes Buyck \& V. Hofstetter (HOLOTYPE: TEXAS. Montgomery Co.: Conroe; PC; MNHN-PC-PC0084085)

Cantharellus lewisii Buyck \& V. Hofstetter (HOLOTYPE: TEXAS. Newton Co.: Bleakwood; PC; MNHN-PC-PC0084074)

Cantharellus tenuithrix Buyck \& V. Hofstetter (HOLOTYPE: TEXAS. Newton Co.: County Road 4045; PC; MNHNPC-PC0084084)

Buyck. B., C. Cruaua, A. Couloux, \& V. Hofstetter. 2011. Cantharellus texensis sp. nov. from Texas, a southern lookalike of C. cinnabarinus revealed by tef-1 sequence data. Mycologia 103(5):1037-1046. (2)

Cantharellus texensis Buyck \& Hofstetter (HOLOTYPE: Texas. Hardin Co.: BTNP, Lance Rosier Unit; PC; MNHNPC-PC0084097)

Buyck, B., I. Olariaga, J. Justice, D. Lewis, W. Roody, \& V. Hofstetter. 2016. The dilemma of species recognition in the field when sequence data are not in phase with phenotypic variability. Cryptog. Mycol. 37(3):367-389. (1)

Cantharellus velutinus Buyck \& V. Hofstetter (HOLOTYPE: TEXAS. Tyler Co.: BTNP, Beech Creek Unit; PC; PC0142227)

Buyck, B., I. Olariaga, B. Looney, J. Justice, \& V. Hofstetter. 2016. Wisconsin chanterelles revisited and first indications for very wide distributions of Cantharellus species in the United States east of the Rocky Mountains. Cryptog. Mycol. 37(3):345-366. (1)

Buyck, B. \& (22 others). 2017. Fungal biodiversity profiles 21-30. Cryptog. Mycol. 38(1):101-146. (1)

Hydnum aerostatisporum Buyck, Lewis, \& Hofstetter (HOLOTYPE: TEXAS. Polk Co.: Big Thicket National Preserve, Big Sandy Creek Unit; PC; PC0142475)

Cooke, W.B. 1961. The genus Schizophyllum. Mycologia 53:575-599. (1)

Cooke, W.B. 1961. The Porotheleaceae, a study of the Cyphelloid Fungi. Behi. Sydowia 4:1-144. (2)

Davoodian, N., K. Hosaka, O. Raspé, O.A. Asher, A.R. Franck, A. de Kesel, T.P. Delaney, J.F. Ammirati, E. Nagasawa, B. Buyck, \& R.E. Halling. 2020. Diversity of Gyroporus (Gyroporaceae, Boletales): rpb2 phylogeny and three new species. Phytotaxa 434(3):208-218. (1)

Desjardin, D.E., S.A. Gordon, \& R.H. Petersen. 1993. Observations on two rhizomorph-forming species of Marasmiellus. Mycol.Res. 97:111-122. (2)

Geesteranus, R.A.M. 1980. The Mycena epipterygia-group. Proc. Kon. Ned. Akad. Wetensch. C. 83(1):65-79. (1)

Gilbertson, R.L. 1964. Resupinate hydnaceous fungi of North America. III. Additional type studies. Pap. Michigan Acad. Sci. 49:15-25. (1)

Gilbertson, R.L. 1981. North American wood-rotting fungi that cause brown rots. Mycotaxon 12:372-416. (12)

Gilbertson, R.L. \& J.L. Lowe. 1970. Tyromyces graminicola in North America. Mycologia 62:699-706. (1)

Halling, R.E. 1983. A synopsis of Marasmius section Globulares. Brittonia 35(4):317-326. (1)

Herald, F.D. \& F.A. Wolf. 1908-1909. The parasitic fungi of Texas. Trans. Texas Acad. Sci. 10-11:10-44. (7) 
Kuo, M. \& B. Ortiz-Santana. 2020. Revision of leccinoid fungi, with emphasis on North American taxa, based on molecular and morphological data. Mycologia 112:197-211. (5)

Lemke, P.A. 1964. The genus Aleurodiscus (sensu lato) in North America. Canad. J. Bot. 42:723-768. (2)

Lentz, P.L. 1955. Stereum and allied genera of fungi in the Upper Mississippi Valley. Agric. Monogr. 24. (4)

Liu, Jian Kui (\& 77 others). 2015. Fungal diversity notes 1-110: Taxonomic and phylogenetic contributions to fungal species. Fungal Diversity 72:1-197. (1)

Russula cortinarioides Buyck, Adamčk, Lewis, \& V. Hofstetter (HOLOTYPE: TEXAS. Newton Co.: Mayflower; PC; PC0142175)

Lloyd, C.G. 1904. Acknowledgment of specimens received from United States and Canada. Puff Ball Letter No. 3, July 1904. (37)

Looney, B.P. 2014. Molecular annotation of type specimens of Russula species described by W.A. Murrill from the southeast United States. Mycotaxon 129(2):255-268. (1)

Lowe, J.L. 1975. Polyporaceae of North America. The genus Tyromyces. Mycotaxon 2:1-82. (1)

Lowe, J.L. \& R. Gilbertson. 1961. Synopsis of the Polyporaceae of the southeastern United States. J. Elisha Mitchell Sci. Soc. 77:43-61. (53)

Mata, J.L., K.W. Hughes, \& R.H. Petersen. 2007. An investigation of Omphalotaceae (Fungi: Euagarics) with emphasis on the genus Gymnopus. Sydowia 58(2):191-289 (3)

Matheny, P.B, R.A. Swenie, A.N. Miller, R.H. Petersen, \& K.W. Hughes. 2018. Revision of pyrophilous taxa of Pholiota described from North America reveals four species - P. brunnescens, P. castanea, P. highlandensis and P. modesta. Mycologia 110(6):997-1016. (1)

McCormick, M.A., M.A. Cubeta, \& L.F. Grand. 2013. Geography and hosts of the wood decay fungi Fomes fasciatus and Fomes fomentarius in the United States of America. N. Amer. Fungi 8(2):1-53. (1)

Methven, A.S. 1990. The genus Clavariadelphus in North America. Biblioth. Mycol. 138:1-192. (2)

Miller, O.K., C.L. Ovrebo, \&W.R. Burk. 1991. Neolysurus: a new genus in the Clathraceae. Mycol. Res. 95(10):1230-1234. (1)

Montoya, L. \& V.M. Bandala. 2005. Revision of Lactarius from Mexico. Additional new records. Persoonia 18(4):471-483.

(1)

Moreno, G., A. Altes, C. Ochoa, \& J.E. Wright. 1995. Contributions to the study of the Tulostomataceae in Baja California, Mexico. Mycologia 87(1):96-120. (2)

Murrill, W.A. 1922. Dark-spored agarics-Il. Gomphidius and Stropharia. Mycologia 14:200-221. (1)

Murrill, W.A. 1922. Dark-spored agarics-III. Agaricus. Mycologia 14:200-221. (1)

Nakasone, K.K. 1990. Cultural studies and identification of wood-inhabiting Corticiaceae and selected Hymenomycetes from North America. Mycol. Mem. 15:1-412. (5)

Nakasone, K.K. 2006. Dendrothele griseocana (Corticiaceae) and related taxa with hyphal pegs. Nova Hedwigia 83:99108. (1)

Neves, M.A. \& R.E. Halling. 2010. Study on species of Phylloporus I: Neotropics and North America. Mycologia 102(4):923-943. (2)

Niskanen, T., K. Liimatainen, J.F. Ammirati, \& K. Hughes. 2013. Cortinarius section Sanguinei in North America. Mycologia 105(2):344-356. (2)

Nuhn, M.E., M. Binder, S.F.S. Taylor, R.E. Halling, \& D.S. Hibbett. 2013. Phylogenetic overview of the Boletineae. Fungal Biology 117:479-511. (3)

Nuytinck, J., S.L. Miller, \& A. Verbeken. 2006. A taxonomical treatment of the North and Central American species in Lactarius sect. Deliciosi. Mycotaxon 96:261-307. (1)

Ortiz-Santana, B., D.P. Lewis, \& E.E. Both. 2009. A new Boletus for North America. Mycotaxon 110:211-217. (1)

Overholts, L.O. 1938. Notes on fungi from the lower Mississippi Valley. Bull. Torrey Bot. Club 65:167-180. (1)

Parker, C.S. 1933. A taxonomic study of the genus Hypholoma in North America. Mycologia 25:160-212. (1)

Pegler, D.N. 1964. A survey of the genus Inonotus (Polyporaceae). Trans. Brit. Mycol. Soc. 47(2):175-195. (2)

Petersen, R.H. 1968 (1971). The genera Gomphus and Gloeocantharellus in North America. Verlag von J. Cramer, Lehre. Pp. 1-118. (1)

Petersen, R.H. 1975. Ramaria subgenus Lentoramaria and emphasis on North America taxa. Biblioth. Mycol. 43:1-161. (1)

Petersen, R.H., D. Desjardin, \& D. Kruger. 2008. Three type specimens designated in Oudemansiella. Fungal Diversity 32:81-96. (1) 
Petersen, R.H. \& K.W. Hughes. 2010. The Xerula/Oudemansiella complex (Agaricales). Nova Hedw. Beih. 137:1-625. J. Cramer, Stuttgart. (4)

Petersen, R.H., K.W. Hughes, J. Justice, \& D.P. Lewis. 2014. A new species of Gomphus from southeastern United States. N. Amer. Fungi 9(9):1-13. (1)

Petersen, R.H. \& K.W. Hughes. 2019. Two Additional Species of Gymnopus (Euagarics, Basidiomycota). MycoKeys 45:1-24. (1)

Petersen, R.H. \& K.W. Hughes. 2020. Two new genera of gymnopoid/marasmioid euagarics. Mycotaxon 135:1-95. (1)

Rea, D.M. 1942. Fungi of southern California I. Mycologia 34:563-574 (1)

Redhead, S.A. 1980. The genus Strobilurus (Agaricales) in Canada with notes on extralimital species. Canad. J. Bot. 58:68-83. (1)

Redhead, S.A. 1989. A biogeographical overview of the Canadian mushroom flora. Canad. J. Bot. 67:3003-3062. (5)

Smith, A.H. 1974. The genus Vascellum (Lycoperdaceae) in the United States. Bull. Mens. Soc. Linn. Soc. Lyon 43:407419. (2)

Vascellum texense A.H. Smith (Holotype: TEXAS. Eastland Co.: Cisco; $\mathrm{MICH} ; 12437$ )

Vascellum intermedium A.H. Smith (HOLOTYPE: TEXAS. Eastland Co.: Cisco; MICH; 12436)

Spirin,V., J. Kout, \& J.Vlasák. 2015. Studies in the Truncospora ohiensis - T. ochroleuca group (Polyporales, Basidiomycota). Nova Hedwigia 100:159-175. (1)

Stubbe, D., J. Nuytinck, \& A. Verbeken. 2010. Critical assessment of the Lactarius gerardii complex (Russulales). Fungal Biology 114:271-283. (3)

Swenie, R.A., T.J. Baroni, \& P.B. Matheny. 2018. Six new species and reports of Hydnum (Cantharellales) from eastern North America. MycoKeys 42:35-72. (2)

Hydnum vagabundum Swenie, Ovrebo, \& Matheny (HOLOTYPE:TEXAS. Newton Co.: Bleakwood; TENN; TENN-F-074443) von Schrenk, H. 1914. Two trunk diseases of the Mesquite. Ann. Missouri Bot. Garden 1:242-252. (1)

Wang, X.-H., R.E. Halling, V. Hofstelter, T. Lebel, \& B. Buyck. 2018. Phylogeny, biogeography and taxonomic re-assessment of Multifurca (Russulaceae, Russulales) using three-locus data. PLOSone 13(11): Nov 7, 2018. (2)

White, V.S. 1901. The Tylostomaceae of North America. Bull. Torrey Bot. Club 28(8):421-444. (1)

Tylostoma albicans V.S. White (HOLOTYPE: TEXAS. Exact location unknown; NY; 00914805; accessioned as Tulostoma)

Wolfe, B.E., M. Kuo, \& A. Pringle. 2012. Amanita thiersii is a saprotrophic fungus expanding its range in the United States. Mycologia 104(1):22-33. (1)

Wright, J.E. 1987. The genus Tulostoma (Gasteromycetes). A world monograph. Biblioth. Mycol. 113:1-338. (13)

Wright, J.E., T. Herrera, \& G. Guzman. 1972. Estudios sobre el genero Tulostoma en Mexico. Ciencia (Mexico) 27:109-122. (1)

Tulostoma meridionale J.E. Wright (HOLOTYPE:TEXAS. Denton Co.: Denton; BPI; BPI 704567)

Wu, Q.-X., G.M. Mueller, F.M. Lutzoni, Y-Q. Huang, \& S-Y. Guo. 2000. Phylogenetic and biogeographic relationships of eastern Asian and eastern North American disjunct Suillus species (Fungi) as inferred from nuclear ribosomal RNA ITS sequences. Molec. Phylogen. Evol. 17(1):37-47. (1)

Zeller, S.M. \& A.H. Smith. 1964. The genus Calvatia in North America. Lloydia 27(3):148-186. (4)

Zeller, S.M. \& C.W. Dodge. 1936. Elasmomyces, Arcangeliella, and Macowanites. Ann. Missouri Bot. Gard. 23:599-638. (1)

\section{DISCUSSION}

Combining the results from this and the previous paper (Lewis \& Ovrebo 2009) there are now 282 journal articles that cite at least one fleshy basidiomycetous collection from Texas. In additions, 103 species or varieties have holotypes designated from the state. We continue to search for records from Texas and suspect that there are more records to be discovered. It is difficult to find records because articles seldom mention the state name in the title so the articles have to be searched individually for citations of Texas collections. These literature records should provide an initial resource to assist mycologists who are undertaking biogeographical, conservation or biodiversity projects. Lewis and Ovrebo (2009) previously listed some of the field guides that include fungi found in Texas. A recently published field guide by Bessette et al. (2019) is an additional valuable resource to help identify all groups of Texas fleshy fungi. 


\section{PART II-NEW RECORDS OF FLESHY BASIDIOMYCOTA FOR TEXAS}

For the listings below the order and family classification as well as specific epithets are those provided in Index Fungorum (http://www.indexfungorum.org/) and Mycobank (www.mycobank.org). It is likely that some of the species below are accessioned in herbaria with older genus or species names; however, we have provided the currently accepted names that mainly result from taxonomic revisions. In this case, searching by collection number might be the best way to locate a collection in a herbarium. Only one record per county is provided but for most counties there may be multiple records that have been made over the years. The fungi reported below are mainly from southeast Texas because that is where most of the collecting has been done by the authors and by those who have forwarded collections to us, but a few records are included from other parts of the state. GPS coordinates are only an indication of the general collection location. Abbreviations for collectors are as follows: DPL-David P. Lewis; CLO-Clark L. Ovrebo. Other abbreviations are as follows: CRCounty Road; FM-Farm to Market Road; SH-State Highway; BTNP-Big Thicket National Preserve.

RESULTS

\section{BASIDIOMYCOTA: AGARICOMYCETES: AGARICALES}

\section{AGARICACEAE}

Battarrea phalloides (Dicks) Pers. Found in arid environments.

Voucher specimen: La Salle Co.: Encinal, 28.0417, -99.3561, Nov 2014, DPL-11658 (TAES).

Echinoderma asperum (Pers.) Bon. Found in mixed pine and hardwood forest.

Voucher specimens: Hardin Co.: BTNP, Lance Rosier Unit, Teel Road at cypress swamp, 30.2643, -94.5125, 12 Nov 2016, DPL-12236 (TAES). Montgomery Co.: Conroe, Graystone Addition, nature trail, 30.3426, -95.4976, 28 Nov 2019, DPL-13457 (TAES). Newton Co.: N of Mayflower, off SH 87, Canyon Rim Nature Trail, 31.1155, -93.7292, 15 Nov 2015, DPL-11891 (TAES). Polk Co: BTNP, Big Sandy Unit, residence by FM 1276, 30.5710, -94.6337, 12 Nov 1989, DPL-4319 (F). San Jacinto Co.: Evergreen, Little Thicket Nature Sanctuary, 30.5416 , $-95.2243,3$ Nov 2018, DPL-13192 (TAES).

Lepiota roseotincta A.H. Smith. Found in mixed pine-hardwood and hardwood forests.

Voucher specimens: Fort Bend Co.: Sugarland, Pepperwood Dr., 29.5846, -95.6109, 28 May 2017, DPL-12384 (TAES). Hardin Co.: Lumberton, Village Creek State Park, nature trail, 30.2545, -94.1710, 30 May 2015, DPL-11747 (TAES). Newton Co.: Bleakwood, CR 3062 \& SH 87, 30.7085, -93.8272, 3 Nov 2015, DPL-11858 (TAES).

Leucoagaricus coerulescens (Peck) J.F. Liang, Zhu L. Yang, \& J. Xu. Found in upland pine forests with oaks. Voucher specimen: Newton Co.: Bleakwood, CR 3062 \& SH 87, 30.7085, -93.8272, 4 Oct 2013, DPL-10953 (TAES).

Leucocoprinus brunnescens (Peck) Pegler. Found in mixed pine and hardwood forests.

Voucher specimens: Hardin Co.: BTNP, Lance Rosier Unit, Teel Road at cypress swamp, 30.2643, -94.5125, 20 Nov 2010, DPL-10065 (TAES). Newton Co.: Bleakwood, CR 3062 \& SH 87, 30.7085, -93.8272, 19 Sep 2009, DPL-9254 (TAES).

Leucocoprinus longistriatus (Peck) H.V. Smith \& N.S. Weber. Found in mixed pine and hardwood forest, duff and flower pots.

Voucher specimens: Hardin Co.: BTNP, Lance Rosier Unit, Cotten Road, 30.2701, -94.5062, 3 Oct 2009, DPL-9388 (TAES). Newton Co.: Bleakwood, CR 3062 \& SH 87, 30.7085, -93.8272, 26 Aug 2016, DPL-12184 (TAES). Orange Co.: BTNP, Beaumont Unit, Houseman Addition, 30.2398, -94.0925, 15 Jul 2014, DPL-11364 (TAES).

\section{AMANITACEAE}

Amanita arkansana Rosen. Found under hardwoods.

Voucher specimens: Newton Co.: Bleakwood, CR 3062 \& SH 87, 30.7075, -93.8280, 24 Sep 2000, DPL 6359 (F). Tyler Co.: Temple-Inland Forest Lake Exp. Forest, 30.6625, -94.1000, 26 Jun 1994, DPL-5302 (RET).

Amanita cinereoconia Atkinson. Found in bottomland hardwoods or mixed hardwood-pine forests. Voucher specimens: Hardin Co.: BTNP, Turkey Creek Unit, Gore Road Trailhead North, 30.522183, -94.34685, 4 Oct 2009, DPL 9416 (TAES). Newton Co.: Bleakwood, CR 3062 \& SH 87, 30.7075, -93.8280, 30 May 2016, DPL-12048 (TAES). Orange Co.: Vidor, off Virginia Lane, 30.1540, -94.0110, 8 Aug 1980, DPL-2228 (F, RET). Polk Co.: BTNP, Big Sandy Unit, 30.6794, -94.6996, 11 Jun 2009, DPL-9062 (TAES). San Jacinto Co.: Evergreen, Little Thicket Nature Sanctuary, 30.5416, -95.2243, 2 Jun 2018, DPL 12875 (TAES). 
Amanita daucipes (Mont.) Lloyd. Found in mixed hardwood or mixed hardwood-pine forests.

Voucher specimens: Hardin Co.: BTNP, Turkey Creek Unit, Kirby Trail, 30.4623, -94.3503, 17 Aug 1980, DPL-2239 (RET). Newton Co.: N of Mayflower, off SH 87, Canyon Rim Trail, 31.1155, -93.7292, 18 Jun 2006, DPL-7444 (FH). Orange Co.: Vidor, Virginia Lane, 30.1535 , -94.0129, 12 Jul 1988, DPL-4136 (F).

Amanita elliptosperma Atkinson. Found in mixed hardwood, pine or pine-hardwood forests.

Voucher specimens: Hardin Co.: BTNP, Lance Rosier Unit, Teel Cemetery, 30.2585, -94.5123, 11 Nov 2009, DPL-9556 (RET). Marion Co.: Caddo Lake Wildlife Management Area, 32.7162, -94.0850, 17 Jun 2017, DPL-12463 (TAES). Newton Co.: Bleakwood, CR 3062 \& SH 87, 30.7075, -93.8280, 12 Aug 2012, DPL-10431(TAES). San Augustine Co.: Angelina Nat. Forest, Turkey Creek Wilderness Area, near Sandy Creek, 31.3469, -94.1894, 25 Oct 1997, DPL-5947 (RET). Walker Co.: Sam Houston National Forest, east side Stubblefield Lake, 30.5597 , -95.6382, 10 Oct 1998, DPL-6092 (RET).

Amanita longipes Bas in Tulloss \& Jenkins. Found in mixed hardwood, pine or pine-hardwood forests. Voucher specimens: Marion Co.: Caddo Lake Wildlife Management Area, 32.7162, -94.0850, 6 Sep 2009, DPL-12615 (TAES). Newton Co.: Bleakwood, CR 3062 \& SH 87, 30.7075, -93.8280, 1 Aug 2009, DPL-9144 (TAES).

Amanita murrilliana Sing. Found under pine.

Voucher specimen: Newton Co.: Bleakwood, CR 3062 \& SH 87, 30.7075, -93.8280, 4 Jul 2008, DPL-8703 (TAES).

Amanita nauseosa (Wakef.) D. Reid. Found on open lawns.

Voucher specimens: Cherokee Co.: Wells, U.S. Hwy. 69, by Dairy Queen, 30 Oct 1998, DPL-6117 (FH, TAES, RET). Galveston Co.: Santa Fe, Cemetery Rd. \& $4^{\text {th }}$ St., 29.4124, -95.1154, 6 Jun 2006, DPL-7459 (FH).

Amanita parcivolvata (Peck) Gilbert. Found in bottomland hardwood forests.

Voucher specimen: Newton Co.: Bleakwood, CR 3062 \& SH 87, 30.7075, -93.8280, 13 Jul 2016, DPL-12139 (TAES).

Amanita pelioma Bas. Found in pine, mixed pine-hardwood or hardwood forests.

Voucher specimens: Hardin Co.: BTNP, Turkey Creek Unit, Kirby Trail, 30.4623, -94.3503, 21 Oct 1984, DPL-3760 (F). Newton Co.: Bleakwood, CR 3062 \& SH 87, 30.7075, -93.8280, 11 Nov 2004, DPL-7084 (TAES). Angelina Co.: Zavalla, Ozias Cemetery, 31.2241, -94.4733, 30 May 1982, DPL-3062 (RET).

Amanita rhoadsii var. rhoadsii (Murr.) Murr. Found in sandy soil in pine-oak forest. Voucher specimen: Newton Co.: Bleakwood, CR $3062 \&$ SH 87, 30.7075, -93.8280, 2 Aug 2014, DPL-11372 (TAES).

Amanita rhopalopus $\mathrm{f}$. rhopalopus Bas. Found in pine forest.

Voucher specimen: Jasper Co.: Evadale, MeadWestvaco Paper Mill, 30.3347, -94.0659, 11 Sep 2000, DPL-6356 (F, RET).

Amanita rhopalopus f. turbinata Bas. Found under hardwoods.

Voucher specimens: Angelina Co.: Zavalla, Dead Man's Rd., CR 317, 31.2123, -91.4433, 17 Nov 2010, DPL-10047 (TAES). Hardin Co.: BTNP, Turkey Creek Unit, Kirby Trail, 30.4623, -94.3503, 12 Aug 1982, DPL-3297 (RET).

Amanita roanokensis Coker. Found on sandy soil beneath pine and oak.

Voucher specimen: Newton Co.: FM 363 \& CR 4045, Sand Ridge Cemetery, 30.7430, -93.7688, 18 Sep 2007, DPL-8284 (RET).

Amanita roseitincta (Murr.) Murr. Found in pine or mixed pine hardwood forests.

Voucher specimens: Hardin Co.: BTNP, Lance Rosier Unit, Teel Road at Cypress Swamp, 30.2643, -94.5125, 8 Jun 2013, DPL-10748 (TAES). Newton Co.: Bleakwood, CR 3062 \& SH 87, 30.7075, -93.8280, 26 Jul 2009, DPL 9141 (TAES). Sabine Co.: Trail Between the Lakes, 31.2678 , -93.8315, 5 Jun 1993, DPL-5001 (BRIT). San Jacinto Co.: Sam Houston National Forest, Big Creek Scenic Area, 30.5074, -95.0903, 10 Jul 1982, DPL-3167 (RET). Tyler Co.: Temple-Inland Forest Lake Experimental Forest, 30.6625, -94.1000, 10 Jun 1995, DPL-5479 (BRIT).

Amanita spreta Peck. Found in mixed pine-hardwood and hardwood forests.

Voucher specimens: Cherokee Co.: South of Rusk at roadside park on U.S. Hwy. 69, 31.7276, -95.1026, 30 Oct 1998, DPL-6119 (F). Hardin Co.: BTNP, Lance Rosier Unit, Teel Road at Cypress swamp, 30.2643, -94.5125, 8 Jun 2013, DPL-10749 (TAES). Jasper Co.: off U.S. Hwy 96 , Hilltop Cemetery, 30.7851, -93.9569, 28 Jun 2017, DPL-12545 (BRIT). Marion Co.: Caddo Lake Wildlife Management Area, 32.7162 , -94.0850, 17 Jun 2017, DPL-12469 (TAES). Newton Co.: Newton, grounds of First United Methodist Church of Newton, 30.8500, -93.7582, 20 Sep 2018, DPL-13096 (TAES).

Amanita subsolitaria Murrill. Found in pine or pine-oak forests.

Voucher specimens: Newton Co.: Bleakwood, CR 3062 \& SH 87, 30.7075, -93.8280, 7 Oct 2007, DPL-8311 (TAES). San Augustine Co.: Angelina Nat. Forest, Turkey Creek Wilderness Area, near Sandy Creek, 31.3469, -94.1894, 25 Oct 1997, DPL-5948 (RET, TAES). San Jacinto Co.: Evergreen, Little Thicket Nature Sanctuary, 30.5416, -95.2243, 2 Jun 2018, DPL-12877 (TAES). Tyler Co.: Temple-Inland Forest Lake Experimental Forest, 30.6471, -94.0978, 9 Jul 2002, DPL-6593 (RET). 
Amanita virginiana (Murr.) Murr. Found under pine and oak.

Voucher specimen: Orange Co.: Vidor, Virginia Lane, 30.1535, -94.0129, 20 Sep 1987, DPL-4079 (F, RET).

\section{CALLISTOSPORACEAE}

Callistosporium purpureomarginatum Fatto \& Bessette. Found on hardwood log. Voucher specimen: Hardin Co.: BTNP, Turkey Creek Unit, Kirby Nature Trail, 30.4623, -94.3503, 2 Jul 2014, DPL-11272 (TAES).

Macrocybe titans (H.E. Bigelow \& Kimbr.) Pegler, Lodge, \& Nakasone. Found in open fields, flower beds and open pine and hardwood forests.

Voucher specimens: Brazos Co.: College Station, Copperfield Road, 30.6499, -96.2938, 14 Sep 2018, DPL-13047 (LSUM). Harris Co.: Humble, Jessie H. Jones Park \& Nature Center, 30.0244, -95.2927, 23 Sep 2014, DPL-11649 (TAES). Montgomery Co.: Conroe, Springwood Glen Lane, 30.3379, -95.4904, 16 Sep 2018, DPL-13088 (LSUM).

\section{CLAVARIACEAE}

Clavaria fragilis Holmsk. Found in pine uplands and beech forest.

Voucher specimens: Hardin Co.: BTNP, Turkey Creek Unit, Kirby Nature Trail, 30.4623, -94.3503, 19 Oct 2013, DPL-10992 (TAES). Tyler Co.: Lake Hyatt, Watson Rare Plant Preserve, 30.5814, -94.3789, 23 Oct 2004, DPL-7003 (F).

Clavulinopsis aurantiocinnabarina Schwein. Found in moist baygall with oaks and beech.

Voucher specimens: Hardin Co.: BTNP, Lance Rosier Unit, Teel Road at Cypress swamp, 30.2643, -94.5125, 10 Jun 2017, DPL-12048 (TAES). Newton Co.: FM $363 \&$ CR 4045, Sand Ridge Cemetery, 30.7430, -93.76878, 11 Jun 2020, CLO-5887 (CSU). San Jacinto Co.: Evergreen, Little Thicket Nature Sanctuary, 30.5416, -95.2243, 3 Nov 2018, DPL-13187 (BRIT).

Clavulinopsis fusiformis (Sowerby) Corner. Found in mixed hardwoods.

Voucher specimens: Newton Co.: Bleakwood, CR 3062 \& SH 87, 30.7075, -93.8280, 16 Nov 2018, DPL-13278 (BRIT). Polk Co.: BTNP, Big Sandy Unit, 30.6148, -94.6709, 29 Nov 1985, DPL-3935 (F). San Jacinto Co.: Evergreen, Little Thicket Nature Sanctuary, 30.5416, -95.2243, 3 Nov 2018, DPL-13188 (BRIT).

Ramariopsis crocea (Peck) Corner. Found in a pine-oak sand hill by a baygall.

Voucher specimen: Newton Co.: FM 363 \& CR 4045, Sand Ridge Cemetery, 30.7430, -93.76878, 30 Dec 2018, DPL-13354 (TAES).

Ramariopsis kunzei (Fr.) Donk. Found in mixed pine, hardwood and beech forest.

Voucher specimens: Hardin Co.: BTNP, Turkey Creek Unit, Kirby Nature Trail, 30.4623, -94.3503, 2 Jul 2014, DPL-11286 (TAES). Polk Co.: BTNP, Big Sandy Unit, Beaver Slide Trail, 30.5732, -94.6425, 4 Jul 2014, DPL-11316 (TAES). Tyler Co.: BTNP, Beech Creek Unit, nature trail, 30.7194, -94.2271, 25 Jun 2014, DPL-11197 (TAES).

\section{CORTINARIACEAE}

Cortinarius bolaris (Pers.) Fr. Found in beech-magnolia-loblolly pine slope forest.

Voucher specimen: Hardin Co.: BTNP, Jack Gore Baygall Unit, ravine area near Arco Oil field road, 30.4788, -94.1682, 28 Nov 1980, DPL$2441(\mathrm{~F})$.

\section{ENTOLOMATACEAE}

Entoloma abortivum (B. \& C.) Donk. Found in mixed pine-hardwood and bottomland hardwood forests. Voucher specimens: Hardin Co.: BTNP, Turkey Creek Unit, Kirby Nature Trail, 30.4623, -94.3503, 25 Oct 2014, DPL-11515 (TAES). Liberty Co.: BTNP, Menard Creek Corridor Unit, 30.4833, -94.8333, 3 Nov 1996, CLO-2984 (CSU). Newton Co.: Bleakwood, CR 3062 \& SH 87, $30.7075,-93.8280,9$ Nov 2014, DPL-11567 (TAES).

Entoloma albidum Murrill. Found in mixed pine-hardwood forests.

Voucher specimen: San Jacinto Co.: Evergreen, Little Thicket Nature Sanctuary, 30.5416, -95.2243, 3 Nov 2018, DPL-13194 (TAES).

Entoloma albinellum (Peck) Hesl. Found in mixed pine-hardwood forest.

Voucher specimen: Hardin Co.: between Kountze and Saratoga, off FM 770, 30.3320, -94.3915, 9 Jun 2018, DPL-12905 (TAES).

Entoloma farlowii (Sing.) Hesl. Found in mixed pine and hardwood forests.

Voucher specimens: Hardin Co.: BTNP, Lance Rosier Unit, Cotten Road, 30.2701, -94.5062, 30 Jun 2014, DPL-11245 (TAES). Orange Co.: Vidor, Our Lady of Lourdes Catholic Church, off FM 105, 30.1481, -94.0153, 13 May 2015, DPL-11701 (TAES).

Entoloma incanum (Fr.) Hesl. Found on a limestone creek embankment with Juniper and Quercus. Voucher specimen: Hays Co.: Wimberley, Blue Hole Regional Park, nature trail, 30.0009, -98.0856, 4 Mar 2017, DPL-12282 (TAES). 
Entoloma murrayi (B. \& C.) Sacc. \& Syd. Found in mixed hardwoods, pine and beech forest.

Voucher specimens: Hardin Co.: BTNP, Lance Rosier Unit, Cotten Road, 30.2701, -94.5062, 8 Jun 2009, DPL-8965 (TAES). Jasper Co.: Erin, residence off FM 1005, 30.8026, -93.9823, 21 Jun 2015, DPL-11809 (TAES). Newton Co.: Bleakwood, CR 3062 \& SH 87, 30.7075, -93.8280, 16 Sep 2009, DPL-9249 (TAES). San Jacinto Co.: Evergreen, Little Thicket Nature Sanctuary, 30.5416, -95.2243, 3 Nov 2018, DPL-13193 (BRIT). Tyler Co.: BTNP, Beech Creek Unit, Nature Trail, 30.7194, -94.2271, 17 Oct 2008, DPL-8738 (TAES).

Entoloma rhodopolium (Fr.) P. Kumm. Found in stream bottomland hardwood forests.

Voucher specimen: Newton Co.: Bleakwood, CR 3062 \& SH 87, 30.7075, -93.8280, 10 Nov 2019, DPL-13426 (TAES).

Entoloma scabrinellum (Peck) Sacc. Found in mixed pine-hardwoods.

Voucher specimen: Newton Co.: N of Mayflower, off SH 87,Canyon Rim Nature Trail, 31.1155, -93.7292, 29 Dec 2018, DPL-13353 (TAES).

Entoloma sinuatum (Bull.) P. Kumm. Found in pine forests.

Voucher specimen: Newton Co.: Bleakwood, CR 3062 \& SH 87, 30.7085, -93.8372, 4 Oct 2013, DPL-10951 (TAES).

\section{HYGROPHORACEAE}

Humidicutis marginata (Peck) Sing. Found in oak, pine and beech forest.

Voucher specimens: Anderson Co.: Elkhart, Ivy Payne Preserve, 31.6302, -95.6009, 26 Mar 1994, DPL-5163 (F). Jasper Co.: Martin Dies State Park, East Loop Trail, 30.8466, -94.1670, 8 Nov 1998, DPL-6152 (F). San Jacinto Co.: Sam Houston National Forest, Big Creek Scenic Area, 30.5074, -95.0903, 28 Apr 2007, DPL-7857 (TAES).

Hygrophorus occidentalis A.H. Smith \& Hesler. Found in mixed pine-hardwood forest.

Voucher specimen: Newton Co.: N of Mayflower, off SH 87, Canyon Rim Nature Trail, 31.1155, -93.7292, 31 Dec 2012, CLO-5083 (CSU).

Hygrophorus pratensis (Fr.) Fr. Found in stream bottomland hardwood forests.

Voucher specimen: Newton Co.: Bleakwood, CR 3062 \& SH 87, 30.7085, -93.8272, 28 Nov 2008, DPL-8826 (TAES).

\section{HYMNOGASTRACEAE}

Gymnopilus fulvosquamulosus Hesler. Found on dead hardwood.

Voucher specimens: Hardin Co.: BTNP, Lance Rosier Unit, Teel Road at cypress swamp, 30.2643, -94.5125, 8 Nov 2014, DPL-11546 (TAES). Jasper Co.: N of Buna, Temple-Inland Black Branch Hunting Club, road 2, 30.5810, -94.0440, 27 Oct 1999, DPL-6206 (F,TAES). Newton Co.: W. of Sabine River off SH 63, 31.0645, -93.5278, 12 Nov 2007, DPL-8313 (TAES).

Gymnopilus liquiritiae (Pers: Fr.) Karst. Found on decaying hardwood logs.

Voucher specimens: Hardin Co.: BTNP, Lance Rosier Unit, Teel Road, 30.2674, -94.5132, 30 Dec 2011, DPL-10278 (TAES). Marion Co.: Lake Caddo Wildlife Management Area, 32.7162, -94.0850, 17 Jun 2017, DPL-12485 (TAES). Newton Co.: Bleakwood, CR 3062 \& SH 87, 30.7085 , -93.8272, 11 Nov 2012, DPL-10547 (TAES). Orange Co.: BTNP, Beaumont Unit, Regal Fern Bog by 4-Oaks Ranch Road, 30.1953, -94.0842, 10 Jun 2009, DPL-9031 (TAES).

\section{INOCYBACEAE}

Inocybe cincinnata (Fr.) Quél. Found in open pine and oak forest.

Voucher specimen: Hardin Co.: Silsbee, Pinecrest Dr., 30.3360, -94.1956, 21 Nov 1996, DPL-5727 (TENN).

Inocybe leptophylla G.F. Atk. Found with upland pines and oak.

Voucher specimen: Tyler Co.: Lake Hyatt, Watson Rare Plant Preserve, 30.5814, -94.3789, 25 Jun 2016, DPL-12107 (TENN).

Inocybe mutata (Peck) Massee. Found in mixed pine and hardwood forest.

Voucher specimen: Hardin Co.: BTNP, Lance Rosier Unit, Teel Road at cypress swamp, 30.2643, -94.5125, 10 Jun 2017, DPL-12430 (TENN).

Inocybe neotropicus Singer, I.J.A. Aguiar, \& Ivory. Found in mixed hardwood bottomlands.

Voucher specimen: Walker Co.: Huntsville, Sam Houston University Field Research Station, 30.7445, -95.4726, 21 Apr 2018, DPL-12843 (SHSU, TENN).

Inocybe rimosa Britzelm. Found under pine.

Voucher specimen: Jasper Co.: Evadale, Temple-Inland Mill, 30.3347, -94.0659, 17 Oct 1993, DPL-5048 (TENN).

Inocybe trechispora (Berk.) P. Karst. Found in mixed pine and hardwood forest.

Voucher specimen: Hardin Co.: Lumberton, Village Creek State Park, nature trail, 30.2545, -94.1710, 30 May 2015, DPL-11738 (TENN).

Inocybe umbrinella Bres. Found in mixed pine and hardwood forest.

Voucher specimen: Hardin Co.: BTNP, Turkey Creek Unit, Gore Road trailhead north, 30.5222 -94.3468, 4 Oct 2009, DPL-9426 (TENN). 
Inosperma lanatodiscum (Kauffman) Matheny \& Esteve-Rav. Found with Quercus virginiana.

Voucher specimen: Orange Co.: Vidor, Our Lady of Lourdes Catholic Church, off FM 105, 30.1481, -94.0153, 27 Nov 2013, DPL-11061 (TENN).

Inosperma rimosoides (Peck) Matheny \& Esteve-Rav. Found in mixed pine and hardwood forests. Voucher specimen: Newton Co.: Bleakwood, CR 3062 \& SH 87, 30.7075, -93.8280, 17 Oct 2012, DPL-10532 (TENN).

\section{LYCOPERDACEAE}

Lycoperdon marginatum Vittad. Found in open pine and hardwood forests and fields.

Voucher specimens: Colorado Co.: Attwater Prairie National Wildlife Refuge, 29.6786, -96.2793, 14 Oct 2011, DPL-10204 (TAES). Hardin Co.: BTNP, Lance Rosier Unit, Teel Road at cypress swamp, 30.2643, -94.5125, 11 Nov 2006, DPL-7679 (TAES). Newton Co.: Bleakwood, CR 3062 \& SH 87, 30.7085, -93.8272, 29 Jul 2006, DPL-7536 (TAES). Tyler Co.: Fred, residence on CR 4900, 30.5493, -94.1747, 6 Aug 1988, DPL4179 (F). Walker Co.: Huntsville, Sam Houston University Field Research Station, 30.7445, -95.4726, 16 Sep 2018, DPL-13077 (TAES).

\section{LYOPHYLLACEAE}

Asterophora lycoperdoides (Bull.) Ditmar. Found on old mushroom likely a Russula in mixed pine-hardwood forest.

Voucher specimen: San Jacinto Co: Sam Houston National Forest, Big Creek Scenic Area, 30.5074, -95.0903, 18 Oct 2003, CLO-4350 (CSU).

\section{MYCENACEAE}

Mycena luteopallens (Pk.) Sacc. Found among hickory detritus.

Voucher specimen: Tyler Co.: BTNP, Turkey Creek Unit, Pitcher Plant Loop Trail, 30.5851, -94.3368, 2 Jul 2014, DPL-11261 (TAES).

Xeromphalina cauticinalis (Fr.) Kühn. Maire. Found in leaf duff in mixed pine and hardwood forest. Voucher specimen: Newton Co.: Bleakwood, CR 3062 \& SH 87, 30.7085, -93.8272, 12 Jan 2018, DPL-12794 (TAES).

\section{OMPHALOTACEAE}

Anthracophyllum lateritium (B. \& C.) Singer. Common on dead hardwood logs and vines.

Voucher specimens: Hardin Co.: BTNP, Lance Rosier Unit, Teel Road at cypress swamp, 30.2643, -94.5125, 9 Jun 2012, DPL-10347 (TAES). Jasper Co.: Erin, residence off FM 1005, 30.8026, -93.9823, 31 Oct 2016, DPL-12219 (TAES). Newton Co.: Bleakwood, CR 3062 and SH 87 , 30.7075, -93.8280, 10 Jun 2012, DPL-10356 (TAES). Orange Co: Vidor, residence off FM 105, 30.1535, -94.0129, 14 May 1980, DPL-2182 (F). Tyler Co.: Ace, 30.5074, -94.8396, 19 Mar 1983, DPL-3454 (F).

Gymnopus iocephalus (B. \& C.) Halling. Found in leaf duff, various forest types.

Voucher specimens: Hardin Co.: BTNP, Lance Rosier Unit, Teel Road at cypress swamp, 30.2643, -94.5125, 7 Jun 2010, DPL-9692 (TAES). Liberty Co.: BTNP, Loblolly Unit, CR 2017, 30.2836, -94.6749, 16 Jun 2008, DPL-8692 (TAES). Newton Co.: Bleakwood, CR 3062 \& SH 87 , 30.7085, -93.8272, 5 Jul 2003, DPL-6741 (F). Orange Co.: BTNP, Beaumont Unit, Houseman Addition, 30.2398, -94.0925, 15 Jul 2014, DPL$11361,(\mathrm{TAES})$.

Gymnopus spongiosus (B. \& C.) Halling. Found in various forest types.

Voucher specimens: Burleson Co.: Caldwell, Jackson Ranch, 30.5605, -96.8350, 27 Jul 2007, DPL-8165 (TAES). Hardin Co.: BTNP, Lance Rosier Unit, Teel Road at cypress swamp, 30.2643, -94.5125, 11 Nov 2006, DPL-7723 (TAES). Hays Co.: Dripping Springs, off SH 12, 30.2534, -98.0560, 26 Feb 2018, DPL-12810 (TAES). Marion Co.: Lake Caddo Wildlife Management Area, 32.7162, -94.0850, 17 Jun 2017, DPL-12486 (TAES). Newton Co.: N of Mayflower, off SH 87, Canyon Rim Nature Trail, 31.1155, -93.7292, 29 Dec 2017, DPL-12765 (BRIT). Orange Co.: Vidor, residence off FM 105, 30.1540, -94.0110, 15 Jan 1989, DPL-4237 (F). Robertson Co.: N of Benchley, Lakeway Dr., 27 Nov 1993, 30.7778, -96.4857, DPL-5138 (TAES). Tyler Co.: BTNP, Turkey Creek Unit, Pitcher Plant Trail, 30.5851, -94.3368, 16 Mar 2014, DPL11115 (TAES).

Lentinula raphanica (Murr.) Mata \& R.H. Petersen. Found on hardwood.

Voucher specimens: Hardin Co.: BTNP, Lance Rosier Unit, Cotten Road, 30.2701, -94.5062, 3 Oct 2009, DPL-9387 (TAES). Newton Co.: Bleakwood, CR 3062 \& SH 87, 30.7075, -93.8280, 20 Jun 2013, DPL-10818 (TAES). Orange Co.: Vidor, off FM 105, 30.1540, -94.0110, 17 May 1977, DPL-957 (F). Polk Co.: BTNP, Big Sandy Unit, Beaver Slide Trail, 30.5732, -94.6425, 11 Jun 2009, DPL-9056 (TAES). Tyler Co.: BTNP, Turkey Creek Unit, Hester Bridge, 30.5606, -94.3340, 2 Jul 2014, DPL-11251 (TAES).

\section{PHYSYLACRIAEAE}

Armillaria gallica Marxm. \& Romagnesi. Found in mixed pine and hardwood forests. Voucher specimens: Newton Co.: N of Mayflower, off SH 87, Canyon Rim Trail, 31.1155, -93.7292, 18 Dec 2007, DPL-8421 (TAES). Montgomery Co.: Sam Houston National Forest, Mitchell Road near SH 149, 30.2892, -95.6847, 28 Dec 2017, DPL-12789 (TAES). Tyler Co.: BTNP, Canyonlands Unit, 30.7476, -94.1516, 20 Dec 2007, DPL-8455 (TAES). 


\section{PLUTEACEAE}

Pluteus atromarginatus (Konrad) Kuhler. Found on hardwoods and sweetbay magnolia.

Voucher specimens: Hardin Co.: BTNP, Lance Rosier Unit, Teel Road at cypress swamp, 30.2643, -94.5125, 8 Nov 2014, DPL-11557 (TAES). Newton Co.: Bleakwood, CR 3062 \& SH 87, 30.7085, -93.8272, 13 Nov 2014, DPL-11578 (TAES).

Volvariella bombycina (Schaeffer: Fries) Sing. Found on various hardwoods.

Voucher specimens: Chambers Co.: Anahuac National Wildlife Refuge, the Willows, 29.6135, -94.5360, 14 Oct 2000, DPL-6369 (LSUM). Galveston Co.: Santa Fe, 29.4205, -95.1164, 30 Jul 2010, DPL-9893 (TAES). Hardin Co.: BTNP, Turkey Creek Unit, Kirby Nature Trail, 30.4623, -94.3503, 4 Oct 2009, DPL-9469 (TAES). Jasper Co.: N of Buna, Temple-Inland Black Branch Hunting Club, road 6, 30.6180 , -94.0206, 19 Sep 1992, DPL-4823 (F). Newton Co.: Bleakwood, CR 3062 \& SH 87, 30.7085, -93.8272, 5 Aug 2016, DPL-12181 (TAES). Tyler Co.: Lake Hyatt, Watson Rare Plant Preserve, 30.5814, -94.3789, 28 Jun 2014, DPL-11229 (TAES)

\section{PTERULACEAE}

Pterula plumosa (Schw.) Fr. Found by decayed hardwood stump.

Voucher specimen: Hardin Co.: Lumberton, Village Creek State Park, nature trail, 30.2545, -94.1710, 30 May 2015, DPL-11752 (TAES).

\section{TRICHOLOMATACEAE}

Tricholoma serratifolium Peck. Found in hardwood forests.

Voucher specimen: Newton Co.: Bleakwood, CR 3062 \& SH 87, 30.7075, -93.8280, 29 Dec 2019, CLO-5877 (CSU).

Tricholomopsis decora (Fr.) Sing. Found on dead wood likely pines.

Voucher specimens: Hardin Co.: Lumberton, Village Creek State Park, 30.2545, -94.1710, 30 May 2015, DPL-11759 (TAES). Newton Co.: Bleakwood, CR 3062 \& SH 87, 30.7075, -93.8280, 29 May 2016, DPL-12047 (TAES). Polk Co.: BTNP, Big Sandy Unit, off FM 1276, 30.5710 , -94.6337, 21 Jun 1986, DPL-3978 (F).

Tricholomopsis formosa (Murr.) Sing. Found on pine stumps and logs.

Voucher specimens: Hardin Co.: BTNP, Old Wagon Road Trail, off FM 2937, 30.4600, -94.1729, 12 Oct 2009, DPL-9507 (TAES). Newton Co.: Trout Creek, E.O. Siecke State Forest, off FR 82, 30.6217, -93.8192, 18 Sep 2018, DPL-13092 (BRIT). Orange Co.: Vidor, Virginia Lane, 30.1540, -94.0110, 29 May 1994, DPL-5203 (F). San Augustine Co.: Turkey Hill Wilderness Area, near FR 307 \& FM 705, 31.3469, -94.1894, 12 Oct 1997, DPL-5936 (TAES). Tyler Co.: BTNP, Turkey Creek Unit, Muscadine Road, 30.5822, -94.3630, 20 Sep 1980, DPL-2325 (F).

\section{TUBARIACEAE}

Flammulaster erinacellus (Peck) Watling. Found on dead hardwoods.

Voucher specimens: Jasper Co.: Erin, residence off FM 1005, 30.8026, -93.9823, 15 May 2017, DPL-12341 (TAES). Marion Co.: Lake Caddo Wildlife Management Area, 32.7162, -94.0850, 6 Sep 2017, DPL-12643 (TAES). Newton Co.: Bleakwood, CR 3062 \& SH 87, 30.7085 , -93.8272, 15 Apr 2015, DPL-11675 (TAES). Polk Co.: BTNP, Big Sandy Unit, corner of FM 943 \& FM 1276, 30.5710, -94.6337, 12 Jun 1976, DPL-234 (TAES). Tyler Co.: BTNP, Canyonlands Unit, Round Lake, 30.6465, -94.0574, 13 Sep 2003, DPL-6803 (F, accessioned as Phaeomarasmius).

\section{BASIDIOMYCOTA: AGARICOMYCETES: INCERTAE SEDIS}

Pseudofistulina radicata (Schw.) Wright and Burdsall. Found in pine uplands with hardwoods. Voucher specimen: Tyler Co.: Lake Hyatt, Watson Rare Plant Preserve, 30.5814, -94.3789, 27 Jun 2015, DPL-11818 (TAES).

\section{BASIDIOMYCOTA: AGARICOMYCETES: BOLETALES}

\section{BOLETACEAE}

Boletus atkinsonii Peck. Found in pine uplands under oaks.

Voucher specimens: Newton Co.: along SH 87, 30.7610, -93.8149, 20 Sep 2018, DPL-13093 (TAES). Polk Co.: BTNP, Big Sandy Unit, Big Sandy Trail, 30.6148, -94.6709, 4 Jul 2014, DPL-11328 (TAES).

Boletus durhamensis B. Ortiz, Bessette, \& McConnell. Found under oaks.

Voucher specimen: Newton Co.: Bleakwood, CR 3062 \& SH 87, 30.7075, -93.8280, 18 Oct 2004, DPL-6953 (CFMR, BUFF).

Boletus fairchildianus (Sing.) Sing. Found in mixed pine-hardwoods.

Voucher specimen: Hardin Co. BTNP, Teel Road at Cypress Swamp, 30.2643, -94.5125, 12 Nov 2013, DPL-12228 (TAES).

Boletus glabellus Peck. Found under live oak.

Voucher specimen: Galveston Co.: Hitchcock, Galveston Memorial Cemetery, 29.3534, -95.0076, 14 Oct 2013, DPL-10980 (TAES). 
Boletus luridellus (Murr.) Murr. Found under live oaks.

Voucher specimens: Galveston Co.: Hitchcock, Mahan Park, 29.3500, -94.9840, 4 Sep 2007, DPL-8242 (TAES). Harris Co.: Houston, Memorial Park, 29.7657, -95.4441, 20 Oct 1984, DPL-3755 (F). Jefferson Co.: Beaumont, Lamar University campus, 30.0452, -94.0765, 26 Jul 1978, DPL-1507 (F). Orange Co.: Doty Road, 30.1401, -93.9475, 13 Sep 1992, DPL-4816 (F). Newton Co.: Newton, near First United Methodist Church of Newton, 30.8500, -93.7581, 20 Sep 2018, DPL-13097 (BRIT).

Boletus pseudoboletinus (Murr.) Murr. Found in sandy soil under mixed pine woods.

Voucher specimens: Brazos Co.: Millican, intersection of HW 6 \& FM 159, 30.4671, -96.2016, 7 Jun 1986, DPL-3969 (F). Hardin Co.: Roy E. Larsen Sandyland Sanctuary, off SH 327, 30.3495, -94.2372, 27 Aug 1986, DPL-4314 (F). Jasper Co.: Evadale, Temple-Eastex Papermill, along canal, 30.3347, -94.0659, 29 Sep 1986, DPL-4024 (F). Newton Co.: Bleakwood, CR 3062 \& SH 87, 30.7075, -93.8280, 2 Jul 2013, DPL10857 (TAES).

Boletus pseudopinophilus A.R. Bessette, Bessette, J. Craine, \& J.L. Frank. Found under pines. Voucher specimen: Jasper Co.: Evadale, MeadWestvaco Paper Mill, 30.3347, -94.0659, 27 Apr 2000, DPL-4816 (TAES).

Boletus purpureorubellus T.J. Baroni, Yetter, \& Norar. Found on well-decayed stump. Voucher specimen: Newton Co.: Bleakwood, CR 3062 \& SH 87, 30.7075, -93.8280, 12 Oct 2004, DPL-6938 (TAES).

Boletus weberi Singer. Found in sandy soil under oaks.

Voucher specimen: Hardin Co.: Roy E. Larsen Sandyland Sanctuary, off SH 327, 30.3495, -94.2372, 6 Jul 1991, DPL-4484 (F).

Buchwaldoboletus sphaerocephalus (Barla) Watling \& T.H. Li. On pine logs or stumps. Voucher specimen: Hardin Co.: BTNP, Jack Gore Baygall Unit, Young Blood Rd, 30.4815, -94.1543, 17 Oct 1993, DPL-5042 (F).

Harrya chromapes (Frost) Halling, Nuhn, Osmundson, \& Manfr. Binder. Found in beech forest. Voucher specimen: Tyler Co.: BTNP, Beech Creek Unit, Beech Woods Trail, 30.7194, -94.2271, 4 Aug 1982, DPL-3242 (F).

Leccinum chalybaeum Singer. Found in mixed pine-oak forests.

Voucher specimens: Hardin Co.: Roy E. Larsen Sandyland Sanctuary, off SH 327, 30.3495, -94.2372, 10 Aug 1982, DPL-3284 (F). Newton Co.: FM 363 \& CR 4045, Sand Ridge Cemetery, 30.7430, -93.7688, 4 Aug 2016, DPL-12172 (TAES).

Neoboletus luridiformis var. pseudosulphureus (Kallenb.) A. Urb. \& Klofac. Found in bottomland hardwoods.

Voucher specimens: Newton Co.: Bleakwood, CR $3062 \&$ SH 87, 30.7075, -93.8280, 16 Sep 2009, DPL-9247 (TAES). Tyler Co.: TempleInland Forest Lake Exp. Forest, 30.6625, -94.1000, 16 Jun 2001, DPL-6470 (F, TAES).

Pulchroboletus rubricitrinus (Murr.) A. Farid \& A.R. Franck. Found under hardwoods or mixed-pine forest.

Voucher specimens: Galveston Co.: Hitchcock, Galveston Memorial Cemetery, 29.3534, -95.0076, 7 Sep 2007, DPL-8263 (TAES). Hardin Co.: BTNP, Teel Road at Cypress swamp, 30.2643, -94.5125, 10 Jun 2017, DPL-12404 (TAES). Jefferson Co.: Beaumont, Pietzsch Elementary School grounds, 30.0440, -94.0851, 26 Jun 1983, DPL-3535 (F). Montgomery Co.: Conroe, North Loop 336, 30.3356, -95.4705, 28 Jul 2007, DPL-8194 (TAES). Tyler Co.: Temple-Inland Forest Lake Exp. Forest, 30.6625, -94.1000, 15 Jun 1994, DPL-5270 (F).

Pulveroboletus curtisii (Berk.) Sing. Found in mixed pine-hardwoods.

Voucher specimens: Hardin Co. BTNP, Lance Rosier Unit, Cotten Road, 30.2701, -94.5062, 1 Sep 1991, DPL-4524 (F). Newton Co.: CR 3062 \&SH 87, 30.7075, -93.8280, 1 Oct 2013, DPL-10930 (TAES). Orange Co.: Vidor, Virginia Lane, 30.1535, -94.0129, 13 Jul 1988, DPL- 4138 (F).

Suillus cothurnatus Singer. Found in pine forests.

Voucher specimens: Hardin Co.: BTNP, Lance Rosier Unit, Teel Rd., 30.2625, -94.5133, 11 Nov 2006, DPL-7710 (TAES). Jasper Co.: N of Buna, Temple-Inland Black Branch Hunting Club, 30.5810, -94.0440, 22 Sep 1991, DPL-4556 (F). Newton Co.: FM 363 \& CR 4045 , Sand Ridge Cemetery, 30.7430, -93.7688, 6 Oct 2010, DPL-13143 (TAES). Tyler Co.: Lake Hyatt, Watson Rare Plant Preserve, 30.5814, -94.3789, 19 Nov 2016, DPL-12253 (TAES).

Tylopilus griseocarneus Wolfe. Found in hardwood or mixed pine-hardwood forests.

Voucher specimens: Cherokee Co.: Rusk, Jim Hogg Park, 31.8047, -95.1259, 27 Jul 2005, DPL-7278 (F). Nacogdoches Co.: Angelina National Forest, Stephen Austin Exp. Forest, 31.5004, -94.7644, 24 Sep 1994, DPL-5342 (F). Polk Co.: BTNP, Big Sandy Unit, Big Sandy Trail, 30.6148, -94.6709, 29 Jul 1978, DPL-1529 (F). San Jacinto Co.: Sam Houston National Forest. 30.5074, -95.0903, 10 Oct 1998, DPL-6108 (TAES, F). Tyler Co.: BTNP, Beech Woods Trail, 4 Aug 1982, DPL-3243 (F).

Tylopilus rubrobrunneus Mazzer \& A.H. Smith. Found in mixed hardwood or hardwood-pine forests. Voucher specimens: Hardin Co.: BTNP, Teel Road at Cypress Swamp, 30.2643, -94.5125, 8 Jun 2013, DPL-10778 (TAES). Jasper Co.: Erin, FM 1005, 30.8026, -93.9823, 15 May 2017, DPL-12346 (TAES). Marion Co.: Caddo Lake Wildlife Management Area, 32.7162, -94.0850, 6 Sep 2017, DPL-12655 (TAES). Newton Co.: Bleakwood, CR 3062 \& SH 87, 30.7075, -93.8280, 26 Sep 2018, DPL-13109 (BRIT). San Jacinto 
Co.: Evergreen, Little Thicket Nature Sanctuary, 30.5416, -95.2243, 2 Jun 2018 DPL-12882 (TAES). Tyler Co.: BTNP, Turkey Creek Unit, 30.5851, -94.3368, 2 Jul 2014, DPL-11263 (TAES).

\section{CONIOPHORACEAE}

Gyrodontium sacchari (Spreng.) Hjortstam. Found on hardwood. New record for U.S.A.

Voucher specimen: Orange Co.: Orange, Shangri La Botanical Garden, 31.1014, -93.7516, 23 Oct 2014, DPL-11509 (TAES).

\section{GOMPHIDIACEAE}

Chroogomphus ochraceus (Kauffman) O.K. Miller. Found under pines.

Voucher specimen: Tyler Co.: BTNP, Canyonlands Unit, 30.7207,-94.1401, 20 Dec 2007, CLO -4726 (CSU, accessioned as C. rutilus (Schaeff.) O.K. Miller).

\section{BASIDIOMYCOTA: AGARICOMYCETES: CANTHARELLALES}

\section{HYDNACEAE}

Clavulina cinerea (Fr.) Schroet. Found in mixed pine and hardwood forest.

Voucher specimens: Hardin Co.: residence off FM 770, 30.3310, -94.3915, 9 Nov 2019, DPL-13406 (TAES). Newton Co.: Bleakwood, CR $3062 \&$ SH 87, 30.7075, -93.8280, 6 Oct 2018, DPL-13130 (BRIT).

Craterellus fallax Smith. Found in mixed pine, hardwood and beech forests.

Voucher specimens: Hardin Co.: BTNP, Jack Gore Baygall Unit, trail off FM 2937, 30.4600, -94.1729, 26 Jul 1985, DPL-3869 (F). Marion Co.: Lake Caddo Wildlife Management Area, 32.7162, -94.0850, 6 Sep 2017, DPL-12624 (TAES). Newton Co.: Bleakwood, CR 3062 \& SH 87 , 30.7075, -93.8280, 10 Nov 2019, DPL-13420 (TAES). Sabine Co.: Yellowpine, Trail Between the Lakes, 31.2678, -93.8315, 5 Jun 1993, DPL$5000(\mathrm{~F})$.

Multiclavula mucida (Pers.) R.H. Peterse. Found among moss on decaying logs.

Voucher specimen: Hardin Co.: BTNP, Lance Rosier Unit, Teel Road at cypress swamp, 30.2643, -94.5125, 7 Jun 2010, DPL-9702 (TAES).

\section{BASIDIOMYCOTA: AGARICOMYCETES: DACRYMYCETALES}

Calocera cornea (Fr.) Loudon. Found on hardwood and pine branches.

Voucher specimens: Hardin Co.: Roy Larsen Sandyland Sanctuary, off SH 327, Nature Trail, 30.3495, -94.2372, 21 Jan 1990, DPL-4333 (F). Polk Co.: BTNP, Big Sandy Unit, Beaver Slide Trail, 30.5732, -94.6425, 4 Jul 2014, DPL-11297 (TAES).

\section{BASIDIOMYCOTA: AGARICOMYCETES: POLYPORALES}

\section{LAETIPORACEAE}

Laetiporus cincinnatus (Morgan) Burds., Banik, \& T.J. Volk. Found on wood.

Voucher specimens: Hardin Co.: BTNP, Turkey Creek Unit, Kirby Nature Trail, 30.4623, -94.3503, 14 Jun 2008, DPL-8657 (TAES). Tyler Co.: BTNP, Canyonlands Unit, 30.7476, -94.1516, 12 Jun 2008, DPL-8626 (TAES).

\section{PODOSCYPHACEAE}

Podoscypha ravenelii (B. \& C.) Pat. Found in mixed pine, hardwood and stream bottomland forests.

Voucher specimens: Hardin Co.: BTNP, Lance Rosier Unit, Teel Road at cypress swamp, 30.2643, -94.5125, 13 Jun 2009, DPL-9114 (TAES). Newton Co.: Bleakwood, CR 3062 \& SH 87, 30.7085, -93.8272, 16 Jun 2018, DPL-12925 (TAES). Polk Co.: BTNP, Big Sandy Unit, Beaver Slide Trail, 30.5732, -94.6425, 11 Jun 2009, DPL-9059 (TAES). Walker Co.: Huntsville, Sam Houston University Field Research Station, $30.7445,-95.4726,16$ Sep 2018, DPL-13082 (TAES).

\section{POLYPORACEAE}

Cryptoporus volvatus (Peck) Shear. Found on Piñon Pine.

Voucher specimen: Brewster Co.: Big Bend National Park, Chisos Mountains at Laguna Meadow, 29.2694, -103.3008, 3 May 1981, DPL-2506 (F).

Favolus tenuiculus P. Beauv. Found on hardwoods.

Voucher specimens: Blanco Co.: Johnson City, Pedernales Oaks Dr., off FM 2766, 30.2912, -98.3334, 1 Nov 2014, DPL-11652 (TAES). Hardin Co.: BTNP, Lance Rosier Unit, Teel Road at cypress swamp, 30.2643, -94.5125, 9 Jun 2012, DPL-10350 (TAES).

Trametes nivosa (Berk.) Murr. Found on beech and other hardwoods.

Voucher specimens: Fort Bend Co.: Brazos Bend State Park, Hale Lake area, 29.3750, -95.5800, 18 Nov 2006, DPL-7757 (TAES). Hardin Co.: BTNP, Turkey Creek Unit, Kirby Nature Trail, 30.4623, -94.3503, 9 May 2017, DPL-12308 (TAES). Newton Co.: N of Mayflower, off SH 87, 
Canyon Rim Nature Trail, 31.1155, -93.7292, 23 Nov 2007, DPL-8352 (TAES). Tyler Co.: BTNP, Canyonlands Unit, 30.7476, -94.1516, 12 Jun 2008, DPL-8637 (TAES).

\section{BASIDIOMYCOTA: AGARICOMYCETES: PHALLALES}

\section{PHALLACEAE}

Blumenavia angolensis (Welw. \& Curr.) Dring. Found on fertile ground or mulch. Voucher specimen: Brazoria Co.: Lake Jackson, approx. 29.0400, -95.4355, 21 Feb 2012, DPL-13534 (TAES).

\section{BASIDIOMYCOTA: AGARICOMYCETES: RUSSULALES}

\section{ALBATRELLACEAE}

Albatrellus cristatus (Schaeff.) Kotl. \& Pouzar. Found in mixed pine-hardwood or hardwood forests. Voucher specimens: Jasper Co.: Erin, off FM 1005, 30.8026, -93.9823, 29 Nov 2013, DPL-11063 (TAES). Newton Co.: Bleakwood, CR 3062 \& SH 87, 30.7075, -93.8280, 24 Nov 2019, DPL-13441 (TAES).

Albatrellus subrubescens (Murr.) Pouzar. Found in mixed pine-hardwood forests. Voucher specimens: Jasper Co.: Erin, off FM 1005, 30.8026, -93.9823, 1 Dec 2013, DPL-11072 (TAES). Newton Co.: N of Mayflower, off SH 87, Canyon Rim Nature Trail, 31.1155, -93.7292, 30 Nov 2009, DPL-9592 (TAES).

\section{HERICIACEAE}

Hericium coralloides (Scop.) Pers. Found on dead oak log. Voucher specimen: Hardin Co.: BTNP, Lance Rosier Unit, Rattlesnake Crossing by SH105, 30.2472, -94.5473, 15 Dec 1979, DPL-2151 (F).

Hericium erinaceus (Bull.) Pers. Found on dead and living Quercus species.

Voucher specimens: Hardin Co.: BTNP, Lance Rosier Unit, Teel Road at cypress swamp, 30.2643, -94.5125, 11 Nov 2006, DPL-7661 (TAES). Henderson Co.: Athens, Athens Botanical Garden, 32.2137, -95.8824, 31 Oct 2004, DPL-7078 (TAES). Liberty Co.: BTNP, Loblolly Unit, 30.2836, -94.6749, 12 Nov 1977, DPL-1226 (F).

\section{RUSSULACEAE}

Lactarius agglutinatus Burlingham. Found in hardwood or mixed pine-hardwood forests.

Voucher specimens: Hardin Co.: BTNP, Turkey Creek Unit, Kirby Trail, 30.4623, -94.3503, 12 May 2017, DPL-11724 (TAES). Newton Co.: Bleakwood, CR 3062 \& SH 87, 30.7075, -93.8280, 23 Oct 2004, DPL-7006 (TAES). Orange Co.: Vidor, Our Lady of Lourdes Catholic Church grounds, 30.1481, -94.0153, 20 May 2015, DPL-12316 (TAES).

Lactarius croceus Burlingham. Found in hardwood and pine-hardwood forests.

Voucher specimens: Galveston Co.: Hitchcock, Galveston Memorial Cemetery, 29.3534, -95.0076, 7 Sep 2007, DPL-8258 (TAES). Hardin Co.: BTNP, Lance Rosier Unit, Teel Road at Cypress Swamp, 30.2643, -94.5125, 10 Jun 2017, DPL-12418 (TAES). Jasper Co.: Erin, off FM 1005, 30.8026, -93.9823, 12 Jun 2017, DPL-12456 (TAES). Marion Co.: Caddo Lake Wildlife Management Area, 32.7162, -94.0850, 17 Jun 2017, DPL-12495 (TAES). Newton Co.: N of Mayflower, off SH 87, Canyon Rim Trail, 31.1155, -93.7292, 29 Jun 2005, DPL-7284 (TAES).

Lactarius lanuginosus Burlingham. Found in mixed pine-hardwood and hardwood forests.

Voucher specimens: San Jacinto Co.: Evergreen, Little Thicket Nature Sanctuary, 30.5416, -95.2243, 3 Nov 2018, DPL-13203 (TAES). Newton Co.: Bleakwood, CR 3062 and SH 87, 30.7075, -93.8280, 10 Nov 2018, DPL-13225 (TAES).

Lactarius neotabidus A.H. Smith. Found under hardwoods-swamp bottomlands.

Voucher specimens: Hardin Co.: BTNP, Lance Rosier Unit, Teel Road at cypress swamp, 30.2643, -94.5125, 7 Jun 2010, DPL-9695 (TAES). Montgomery Co.: Conroe, Dogwood Dr., 30.3910, -95.2921, 18 Aug 2012, DPL-10433 (TAES). Newton Co.: Bleakwood, CR 3062 \& SH 87 , 30.7075, -93.8280, 6 Oct 2018, DPL-13135 (BRIT). Orange Co.: Vidor, Virginia Lane (swamp area), 30.1540, -94.0110, 9 Jun 1977, DPL-1031 (F). Polk Co.: BTNP, Menard Creek Corridor Unit, Holly Grove area, 30.5036, -94.6886, 26 April 2010, DPL-9633 (TAES).

Russula perlactea Murr. Found in mixed pine, hardwood and beech forests.

Voucher specimens: Hardin Co.: BTNP, Lance Rosier Unit, Teel Road at cypress swamp, 30.2643, -94.5125, 16 Nov 2013, DPL-11047 (TAES). Newton Co.: Bleakwood, CR 3062 \& SH 87, 30.7085, -93.8272, 1 Jan 2007, DPL-7804 (TAES). Tyler Co.: BTNP, Canyonlands Unit, 30.7207 , -94.1401, 20 Dec 2007, DPL-8448 (TAES).

\section{BASIDIOMYCOTA: AGARICOMYCETES: THELEPHORALES}

\section{BANKERACEAE}

Hydnellum concrescens (Pers.) Banker. Found in mixed pine-hardwood forest.

Voucher specimens: Jasper Co.: Erin, residence off FM 1005, 30.8026, -93.9823, 9 Nov 2018, DPL-13232 (TAES). Newton Co.: Bleakwood, 
CR 3062 \& SH 87, 30.7085, -93.8280, 9 Oct 2018, DPL-13170 (TAES). San Jacinto Co.: Evergreen, Little Thicket Nature Sanctuary, 30.5416 , -95.2243, 3 Nov 2018, DPL-13213 (TAES).

Hydnellum spongiosipes (Peck) Pouzar. Found in mixed pine-hardwood forest.

Voucher specimen: Hardin Co.: BTNP, Lance Rosier Unit, Teel Road at Cypress Swamp, 30.2643, -94.5125, 11 Jun 2016, DPL-12070 (TAES).

Phellodon alboniger (Peck) Banker. Found in mixed pine-hardwoods.

Voucher specimen: Tyler Co.: Lake Hyatt, Watson Rare Plant Preserve, 30.5814, -94.3789, 10 Nov 2018, DPL 13241 (TAES).

Phellodon confluens (Pers.) Pouzar. Found in hardwood or mixed pine-hardwood forests.

Voucher specimens: Newton Co.: Bleakwood, CR 3062 \& SH 87, 30.7075, -93.8280, 1 Oct 2018, DPL-13120 (TAES). Jasper Co.: Erin, residence off FM 1005, 30.8026, -93.9823, 9 Nov 2018, DPL-13234 (TAES).

Sarcodon atroviridis (Morgan) Banker. Found in mixed pine-oak forest.

Voucher specimen: Newton Co.: Bleakwood, CR 3062 \& SH 87, 30.7075, -93.8280, 13 Oct 2018, DPL-13174 (TAES).

Sarcodon underwoodii Banker. Found in mixed pine-hardwoods.

Voucher specimen: Tyler Co.: Lake Hyatt, Watson Rare Plant Preserve, 30.5814, -94.3789, 10 Nov 2018, DPL-13249 (TAES)

\section{THELEPHORACEAE}

Thelephora multipartita Schwein. Found on soil.

Voucher specimen: Polk Co.: BTNP, Big Sandy Creek Unit, Beaver Slide Trail, 30.5732, -94.6425, 11 Jun 2009, DPL-9060 (TAES).

Thelephora vialis Schwein. Found under hardwoods.

Voucher specimens: Hardin Co.: BTNP, Turkey Creek Unit, Kirby Trail, 30.4623, -94.3503, 22 Jul 2007, DPL-8140 (TAES). Tyler Co.: Temple-Inland Forest Lake Exp. Forest, 30.6625, -94.1000, 16 Jun 2001, DPL-6471 (LSUM). Newton Co.: Bleakwood, CR 3062 \& SH 87, 30.7075, -93.8280, 26 Sep 2018, DPL-13107 (BRIT).

\section{BASIDIOMYCOTA: TREMELLOMYCES: TREMELLALES}

\section{TREMELLACEAE}

Phaeotremella foliacea (Pers.) Wedin, J.C. Zamora, \& Millanes (accessioned as Tremella foliacea Persoon: Fries.) Found on dead hardwood.

Voucher specimens: Hardin Co.: BTNP, Lance Rosier Unit, Teel Road at cypress swamp, 30.2643, -94.5125, 14 Jun 2014, DPL-11173 (TAES). Tyler Co.: BTNP, Canyonlands Unit, off FM 92, 30.7476, -94.1516, 9 Jun 2010, DPL-9780 (TAES).

Tremella fuciformis Berkeley. Found on hardwood logs and branches.

Voucher specimens: Hardin Co.: Roy E. Larsen Sandyland Sanctuary, off SH 327, 30.3495, -94.2372, 17 Sep 1983, DPL-3599 (F). Hays Co.: Dripping Springs, 30.2534, -98.0560, 26 Feb 2018, DPL-12813 (TAES). Newton Co.: Bleakwood, CR 3062 \& SH 87, 30.7075, -93.8280, 11 Oct 2009, DPL-9484 (TAES).

\section{DISCUSSION}

The 120 new Texas species records reported here as well as the 52 records previously published (Lewis \& Ovrebo 2009) are an indication of the diversity of fleshy basidiomycetous fungi for a still largely underexplored region of the U.S.A. The diversity of forest and habitat types in southeast Texas and Texas as a whole will continue to yield many more new records and likely new species. Progress is slow in documenting mushroom diversity for several reasons: the unpredictability of their fruiting patterns which is dependent on sufficient rainfall; the ephemeral nature of the basidiomata—they appear for only a short time; and because of the lack of naturalists or biologists who are willing to devote time to collecting them. Most all field guides give instructions on how to collect and preserve the mushroom-type fungi and so both amateurs as well as professionals can help advance our knowledge of their diversity. As was justified above for publishing journal article records for Texas fungi, the vouchered records listed here as well as those in Lewis and Ovrebo (2009) will likewise provide additional data for biogeographical or biodiversity studies and provide a source for herbaria from which specimens can be borrowed. 


\section{ACKNOWLEDGMENTS}

Thanks are extended to the Big Thicket National Preserve for granting permission to collect in the Big Thicket. Rodham Tulloss and Brandon Matheny kindly provided assistance with identifications of Amanita and Inocybe species, respectively. Juan L. Mata and Harold W. Keller are thanked for providing constructive reviews of the manuscript. We also extend thanks to the many authors who have published on taxa from Texas and many amateurs who have forwarded collections to us including Nancy Greig, Deborah Mersky, Jolyn Piercy, Paul Roling, Tim Taroni, and David Werth.

\section{REFERENCES}

LeWIS, D.P. \& C.L. OvreBo. 2009. Mycological literature on Texas fleshy Basidiomycota, two new combinations, and new fungal records for Texas. J. Bot. Res. Inst. Texas 3(1):257-271.

Bessette, A.E., A.R.Bessette, \& D.P. Lewis. 2019. Mushrooms of the Gulf Coast States. A field guide to Texas, Louisiana, Mississippi, Alabama, and Florida. University of Texas Press, Austin, Texas, U.S.A. 